\title{
F-TOUCH Sensor for Three-Axis Forces Measurement and Geometry Observation
}

\author{
Wanlin $\mathrm{Li}^{1}$, Yohan $\mathrm{Noh}^{3}$, Akram Alomainy ${ }^{2}$, Ivan Vitanov ${ }^{1}$, Yu Zheng ${ }^{4}$, Peng $\mathrm{Qi}^{5}$, Kaspar Althoefer ${ }^{1}$ \\ ${ }^{1}$ Centre for Advanced Robotics @ Queen Mary (ARQ), Queen Mary University of London, London, UK \\ ${ }^{2}$ School of Electronic Engineering and Computer Science, Queen Mary University of London, London, UK \\ ${ }^{3}$ Department of Mechanical and Aerospace Engineering, Brunel University London, London, UK \\ ${ }^{4}$ Tencent Robotics X, Shenzhen, China \\ ${ }^{5}$ Department of Control Science and Engineering, Tongji University, Shanghai, China
}

\begin{abstract}
We present an innovative concept for a sensor design that can simultaneously measure multi-axis force information and acquire geometry information with the use of a vision-based technique. The sensor is named F-TOUCH (force and tactile optically unified coherent haptics) which is originally inspired by the GelSight tactile sensor. However, the GelSight tactile sensor uses numerous markers painted between the coating layer and the elastomer base, and it can not well generalize the force-related information from the GelSight images [1]. The F-TOUCH sensor is enhanced with a three-axis force measurement capability by virtue of an internal elastic structure placed underneath the entire elastomer layer, as well as using a conventional force sensor calibration method. The proposed sensor uses a camera to concurrently record the mechanical deformation of the elastic structure (for normal and shear forces measurement) and the surface distortion of the elastomer layer (for geometry observation). Results show that the F-TOUCH sensor is effective in generalizing the forcerelated information from the images and performing brilliant multi-axis force measurements (comparing with a commercial force sensor), as well as capturing the object's geometry at the same time.
\end{abstract}

\section{Keywords-Force sensor; tactile sensing; robotics}

\section{INTRODUCTION}

It is well accepted that the acquisition of high-quality force and tactile sensor signals during a mechanical interaction between a robot and an object provides the opportunity to largely enhance the handling and manipulation capability of the robotic system. Deploying force sensors and tactile sensors within robot end-effectors has improved the object grasping, obstacle avoidance, in-hand manipulation, and interactions with the external environment [1-4]. Artificial force and tactile sensing system for robot end-effector [5] has been proposed during the past few decades. The core concept is to combine different sensors and let them work together as an integrated system to perform force and tactile sensing. A typical layout is to apply tactile sensors at the end for tactile perception and force sensors between the end and joint for force measurement

Currently, there is a clear distinction between a typical force sensor and a tactile sensor. On one hand, multi-axis force sensors are developed to measure forces and torques that occur in more than one direction within the robotic force and tactile sensing system. Force sensors usually include a mechanical structure and by sensing the deformation of the structure under external load, multi-axis force/torque (F/T) quantities can be measured along with different directions. Current popular sensing methods include strain sensing technology (semiconductor strain gauges [6], capacitive strain gauging [7], fiber bragg gratings [8], and electrical resistance strain gauges [9]), optical-based sensing technology [10], etc. Mechanical structures include cross-beam structure [11], Stewart platform [12], cantilever structure [13] and spring structure [14] etc. On the other hand, there has been a surge of research interest in tactile sensor development in recent decades. Different technologies [15] have been explored to acquire tactile information that includes mechanical stimulus detection of contact, slip, pressure, geometry, etc. Typical technologies are electrical-based (piezoresistive, piezoelectric, capacitive, and magnetic) and optical-based [16]. Therefore, force sensors can only measure touchpoint multi-axis force/torque components without tactile perception (for example geometry or distribution), while tactile sensors can perceive tactile information but cannot preciously measure multi-axis $\mathrm{F} / \mathrm{T}$ components.

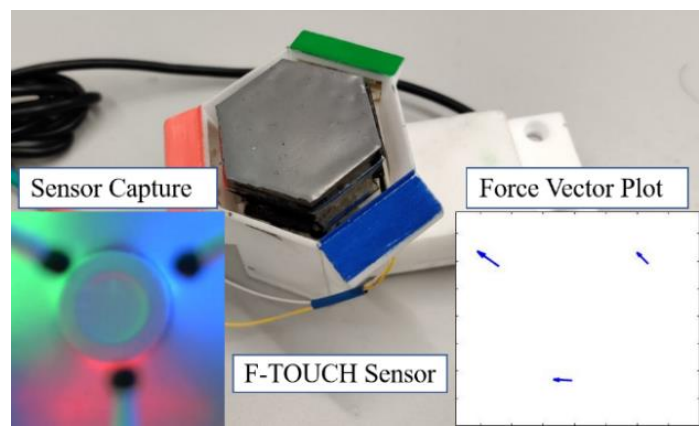

Fig. 1. The manufactured prototype of the F-TOUCH sensor. The sensor is connected via a USB webcam to capture real-time data (both force information and tactile information). An internal spring-mechanism structure with black markers is used to measure the three-axis force.

Our contribution in this paper is to integrate the capabilities of both a force sensor and a tactile sensor into one sensor structure. The sensing principle is based on an optical method that uses a single vision transducer (a camera) to sense both force and tactile modalities. The proposed sensor is named F-TOUCH (force and tactile optically unified coherent haptics) which is shown in Fig. 1.

\section{SENSOR DESIGN AND IMPLEMENTATION}

As is shown in Fig. 2, the overall design of the F-TOUCH sensor is composed of three main parts: coated elastomer (1) (section $\mathrm{A}$ ) reveals the tactile information as well as isolating the ambient light interference; elastomer holder (2), springs (3), magnets (4), force marker (5) make up the springmechanism structure (section $\mathrm{B}$ ), which estimates the force information; A Logitech C920 webcam (6) and SMD LED arrays (7) are used for data capture and illumination. 


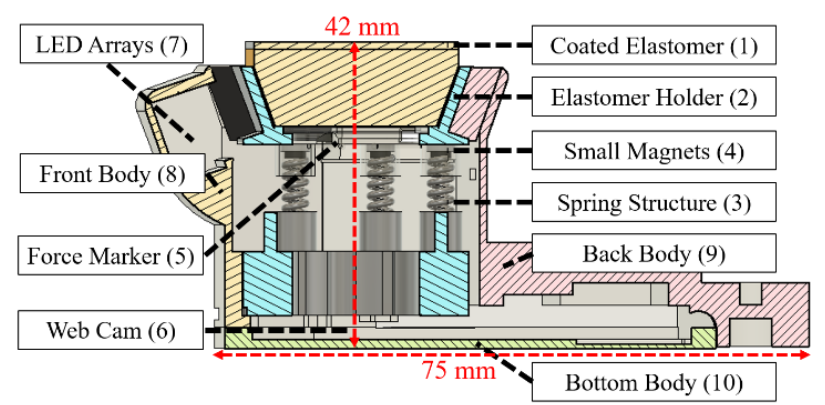

Fig. 2. Schematic design of the F-TOUCH sensor. There are in total of 10 components that make up the device. Force marker (5) and small magnets (4) will be described in detail in Fig. 4.

\section{A. Fabrication of Silicone Elastomer Layer}

The coated transparent elastomer layer is a core part of FTOUCH sensor design, as the elastomer's properties largely affect the sensitivity of the sensor. The elastomer layer is composed of two parts: a transparent silicone base and a reflective coating membrane. An appropriate elastomer base needs to be of the appropriate hardness and elasticity, and the membrane surface needs to be uniform, thin, and smooth. We follow the instruction in [17] and the produced elastomer layer (with a hardness of Shore A 15) can reveal the object's geometry during contact (shown in Fig. 5).

\section{B. Fabrication of Spring Mechanism Structure}

The internal spring mechanism structure is placed under the silicone elastomer (shown in Fig. 4). This elastic structure contains a 3D printed elastomer holder (in VeroClear material); 6 compression springs (15 mm length, $4 \mathrm{~mm}$ in diameter, and of $0.5 \mathrm{~mm}$ wire diameter); 24 magnets ( $2 \mathrm{~mm}$ height, of $3 \mathrm{~mm}$ and $4 \mathrm{~mm}$ diameters); three spherical markers (painted into black) to measure the three-axis force components; and a 3D printed bottom platform (in Nylon material). The use of the springs ensures the linearity of the mechanical structure and the use of the magnets connection stabilizes the overall structure, thus increases the structure's robustness and also reduces the hysteresis.

The movements of the black markers are coupled with the deformation of the springs, and the plane made up by the three markers is always in parallel with the bottom surface of the elastomer layer. During the interaction with an object, the physical contact along the sensing medium leads to a compression of the six springs and results in a movement of the upper platform plane (contains the three markers). We used a camera underneath the structure to capture both the movement of each marker and the area changes. We choose the markers to be black so that markers are easily tracked and segmented from the RGB tactile image. Since the spring constant is relatively low compared with the hardness of the silicone elastomer layer, the spring-mechanism structure will move as a whole body together with the elastomer layer when an external load is applied.

\section{SENSOR EVALUATION AND CALIBRATION}

To evaluate the performance of F-TOUCH sensor, we analyze from four steps: force and tactile information segmentation, calibration for normal force and shear force, normal force and shear force evaluation, and simultaneous force measurement and tactile perception.

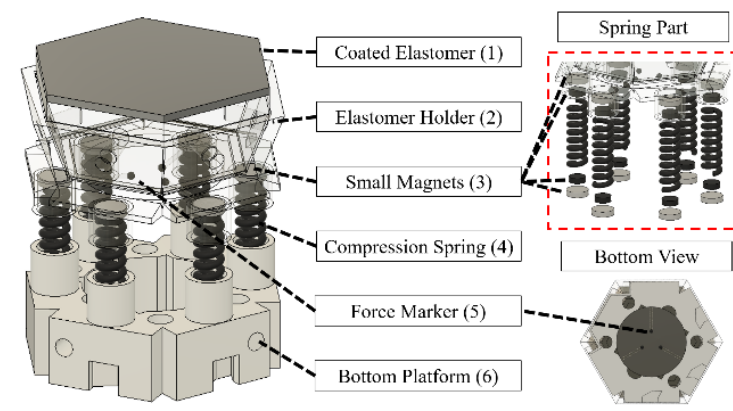

Fig. 4. CAD drawing of the proposed spring-mechanism structure which is made of five components shown as (2) to (6). The top section is composed of the elastomer holder which has 6 magnets glued at its downward plane. The middle section includes 6 springs with 12 magnets glued on both ends. The bottom platform has 6 magnets glued on its upward plane.

\section{A. Force and Tactile Information Segmentation}

When the sensor is on, RGB lights are emitted from different directions to illuminate our sensing system (for both coated elastomer surface and black markers within the spring structure). When there is a contact occurring on the surface, the raw image is received by the camera at the bottom. The procedure of segmenting force information and tactile information within the raw image doesn't require massive computation. Since there is a distinct color difference between the contact geometry (only red, green, blue color can be observed) and the black marker, we firstly transfer the raw image into a grayscale image and then apply a gaussian filter. After the filtering, we apply a threshold to the filtered grayscale image to segment the marker areas from the background and then conduct a color reverse (see Fig. 5).
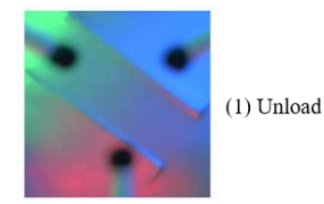

Raw Image

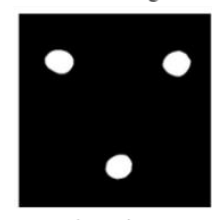

Binary image
Grayscale

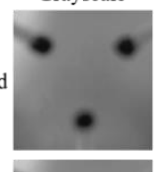

(2) $-\mathrm{Fx}$

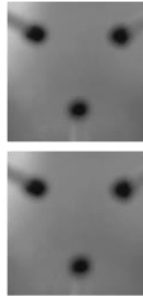

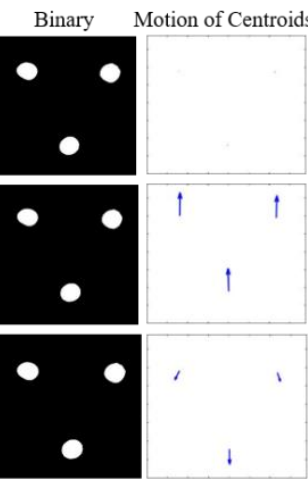

Fig. 5. Force information segmentation: The top left is the raw capture, Bottom left is the binary image after filtering and thresholding. The right part shows the sensor's responses under different force exertion in the form of the grayscale image, reverse binary image and the motion of each marker centroids in the vector plot. A scale is multiplied to the marker's centroid movements therefore motions are enhanced for better observations.

\section{B. Calibration for Normal Force and Shear Force}

After the force information segmentation, a calibration process is needed for normal and shear force. This process aims to obtain a relationship between the nine variables outputs of F-TOUCH sensor (change of $x, y$ coordinates of three markers' centroids from the binary image and the corresponding area changes) and a commercial sensor's input forces. To set up the experiment, we use ATI Mini40 six-axis F/T sensor as the ground-truth. Then we mount our sensor on top of ATI sensor via a 3D printed connector. We manually apply different force conditions (see Table I) on the touch 
medium of our sensor, as shown in Fig. 6. We synchronize both readings from our sensor and the ATI sensor and record a set of ground truths force data of 8400 samples together with marker's centroids and areas change readings from our sensor in a response rate of 15 samples per second (the camera frame rate is $30 \mathrm{fps}$ ). From the recorded data, a calibration matrix can be calculated using the least squares regression method [18].

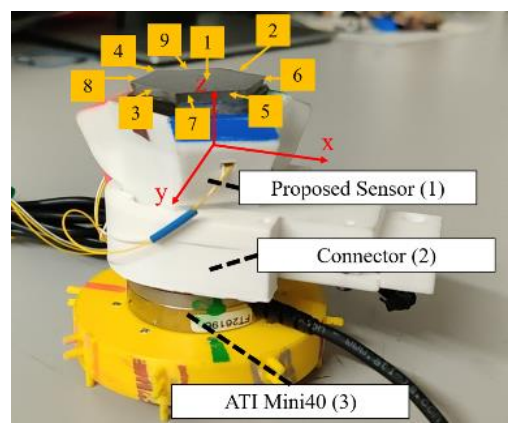

Fig. 6. Experimental setups for calibration of three-axis forces. Nine force conditions are applied to the elastomer surface of the F-TOUCH sensor.

We assume that the nine-variables outputs (marker centroids $X, Y$ coordinate and area $A$ changes) of our sensor are independent, namely $X_{1}, Y_{1}, A_{1}, X_{2}, Y_{2}, A_{2}, X_{3}, Y_{3}, A_{3}$, and the applied force $F x F y F z$ are dependent variables. We can generate a 3-by-9 calibration matrix $K$. This matrix converts nine sensor readings to three physical values of forces. The calculated calibration matrix $K$ is presented in Eq. 1 and 2.

$$
\left[\begin{array}{lllllllll}
k_{11} & k_{12} & k_{13} & k_{14} & k_{15} & k_{16} & k_{17} & k_{18} & k_{19} \\
k_{21} & k_{22} & k_{23} & k_{24} & k_{25} & k_{26} & k_{27} & k_{28} & k_{29} \\
k_{31} & k_{32} & k_{33} & k_{34} & k_{35} & k_{36} & k_{37} & k_{38} & k_{39}
\end{array}\right] \cdot\left[\begin{array}{c}
X_{1} \\
Y_{1} \\
A_{1} \\
X_{2} \\
Y_{2} \\
A_{2} \\
X_{3} \\
Y_{3} \\
A_{3}
\end{array}\right]=\left[\begin{array}{c}
F x \\
F y \\
F z
\end{array}\right]
$$

TABLE I. APPLIED ForCE CONDITIONS ON SENSORS

\begin{tabular}{|c|c|c|c|}
\hline Applied force & Condition & Applied force & Condition \\
\hline 1 & $-F z \pm F x \pm F y$ & 6 & $+F y,-F x$ \\
\hline 2 & $+F y$ & 7 & $-F y,-F x$ \\
\hline 3 & $-F y$ & 8 & $-F y,+F x$ \\
\hline 4 & $+F x$ & 9 & $+F y,+F x$ \\
\hline 5 & $-F x$ & & \\
\hline
\end{tabular}

$K=\left[\begin{array}{ccccccccc}-9.3 e^{-4} & -2.0 e^{-3} & 4.5 e^{-4} & 3.6 e^{-3} & -1.9 e^{-3} & -3.8 e^{-4} & -2.0 e^{-3} & 2.8 e^{-4} & -1.8 e^{-4} \\ 60 e^{-3} & -2.9 e^{-3} & -1.5 e^{-3} & -3.5 e^{-3} & 4.1 e^{-3} & -1.2 e^{-3} & 1.3 e^{-3} & -2.0 e^{-3} & -2.2 e^{-3} \\ 3.0 e^{-2} & -3.6 e^{-2} & 5.5 e^{-3} & 5.7 e^{-3} & 63 e^{-2} & -1.9 e^{-3} & -3.5 e^{-2} & -2.8 e^{-2} & -4.2 e^{-4}\end{array}\right]$

$K=\left[\begin{array}{ccccccccc}6.0 e^{-3} & -2.9 e^{-3} & -1.5 e^{-3} & -3.5 e^{-3} & 4.1 e^{-3} & -1.2 e^{-3} & 1.3 e^{-3} & -2.0 e^{-3} & -2.2 e^{-3} \\ 3.0 e^{-2} & -3.6 e^{-2} & 5.5 e^{-3} & 5.7 e^{-3} & 6.3 e^{-2} & -1.9 e^{-3} & -3.5 e^{-2} & -2.8 e^{-2} & -4.2 e^{-4}\end{array}\right]$

\section{Normal Force and Shear Force Evaluation}

The estimated three-axis force $F x F y F z$ can be obtained by multiplying the calibration matrix $K$ with the nine outputs of our sensor. To evaluate the calibration matrix, external force components are applied on the membrane of our sensor under two force conditions (apply shear force to the elastomer with almost zero normal force and apply normal force plus shear force to the elastomer). We compare our sensor force reading with the ground-truth values from ATI Mini40, as is shown in Fig. 7 and Table II. The root mean square error (RMSE) of $F x F y ~ F z$ is $0.04 \mathrm{~N}, 0.05 \mathrm{~N}$, and $0.13 \mathrm{~N}$, respectively, which is superior to the GelSight performance of $0.187 \mathrm{~N}, 0.162 \mathrm{~N}$, and $0.668 \mathrm{~N}$, respectively [17].

TABLE II. PROPOSED SENSOR ForCE MEASUREMENT ACCURACY

\begin{tabular}{|c|c|c|c|}
\hline Force & Measure range & Maximum error & RMSE \\
\hline$F x$ & $+/-1 \mathrm{~N}$ & $11.97 \%$ & $0.04 \mathrm{~N}$ \\
\hline$F y$ & $+/-1 \mathrm{~N}$ & $15.93 \%$ & $0.05 \mathrm{~N}$ \\
\hline$F z$ & $0-8 \mathrm{~N}$ & $8.77 \%$ & $0.13 \mathrm{~N}$ \\
\hline
\end{tabular}

\section{CONCLUSION AND FUTURE WORK}

In this paper, we have introduced a vision-based sensor named F-TOUCH that uses a combination of a coated silicone elastomer and a spring-mechanism structure. The proposed sensor can preciously measure three-axis force components (with better performance than the GelSight tactile sensor) and observe the geometry information at the same time. The concept of sensing two modalities within one sensor can be useful when it is connected to a robot end effector to provide sufficient force and tactile feedback without complex integration. More work will focus on providing an accurate measurement of torques, as well as exploring the tactile information aspect in terms of texture classification.

\section{ACKNOWLEDGMENT}

This work was financially supported by the EPSRC National Centre for Nuclear Robotics project $(\mathrm{EP} / \mathrm{R} 02572 \mathrm{X} / 1)$, the Innovate UK WormBot project (104059), the CCF-Tencent Open Fund (RAGR20190120), the National Natural Science Foundation of China under Grant 51905379, and Zhejiang Lab's International Talent Fund for Young Professionals.
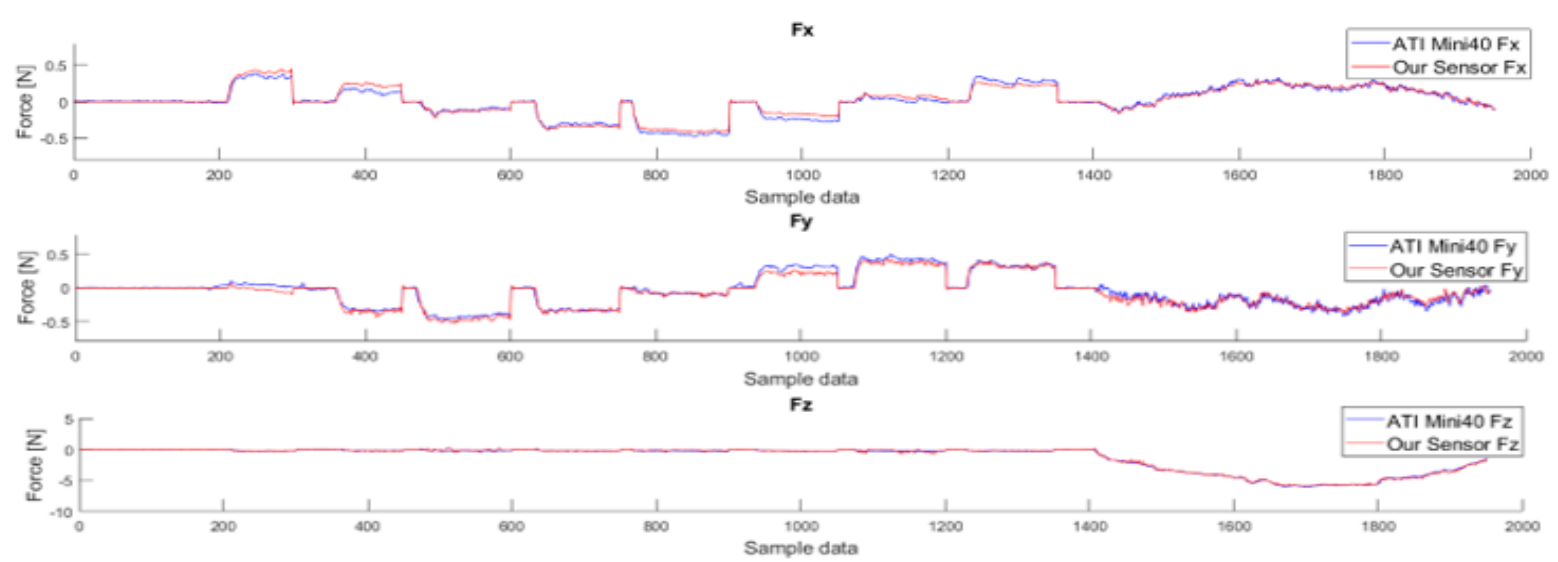

Fig. 7 Three-axis force comparison between ATI Mini40 sensor and F-TOUCH sensor. The first stage is applying pure shear force to the elastomer (normal force is tiny and almost to be zero, this can be achieved by the spring-structure) and the second stage is applying normal force plus shear force. 


\section{REFERENCES}

[1] M. Ohka, Y. Mitsuya, I. Higashioka and H. Kabeshita, "An experimental optical three-axis tactile sensor for micro-robots", in Robotica 23, 457-465, 2005.

[2] Y. Noh, L. Lindenroth, S. Wang, R. Housden, A. Wingerden, W. Li and K. Rhode, "A 2-piece six-axis force/torque sensor capable of measuring loads applied to tools of complex shapes", in Proceedings of 2019 IEEE/RSJ International Conference on Intelligent Robots and Systems (IROS), 2019.

[3] Y. Noh, J. Bimbo, S. Sareh, H. Wurdemann, J. Fras, D. S. Chathuranga, H. Liu, J. Housden, K. Althoefer and K. Rhode, "Multi-axis force/torque sensor based on simply-supported beam and optoelectronics", in Sensors 2016, vol. 16, no. 11, p.1936, 2016.

[4] H Xie, A Jiang, L Seneviratne and K Althoefer. "Pixel-based optical fiber tactile force sensor for robot manipulation", in Proceedings of the 2012 IEEE Sensors, pp. 1-4, 2012.

[5] R. D. Howe, "Tactile sensing and control of robotic manipulation", in Advanced Robotics, 8(3):245-261, 1993.

[6] H.-P. Phan, D. V. Dao, K. Nakamura, S. Dimitrijev, and N.-T. Nguyen, "The piezoresistiveeffect of sic for mems sensors at high temperatures: a review", in Journal of Microelectrome-chanical systems, 24(6):16631677, 2015.

[7] A.L. Trejos, R.V. Patel, and M.D. Naish, "Force sensing and its application in minimally invasive surgery and therapy: a survey", in Proceedings of the Institution of Mechanical Engineers, Part C: Journal of Mechanical Engineering Science 224.7 (2010): 1435-1454, 2010.

[8] K. T. V. Grattan and T. Sun, "Fiber optic sensor technology: An overview", Sens. Actuators, vol. 82, pp. 40-61, 2000.

[9] W. M. Murray and W. R. Miller, "The Bonded Electrical Resistance Strain Gage: An Introduction”, in U.K., London:Oxford Univ. Press, 1992.

[10] O. Al-Mai, M. Ahmadi and J. Albert, "Design, Development and Calibration of a Lightweight, Compliant Six-Axis Optical
Force/Torque Sensor," in IEEE Sensors Journal, vol. 18, no. 17, pp. 7005-7014, 2018

[11] L. Xiong, G. Jiang, Y. Guo, H. Liu, “A three-dimensional Fiber bragg grating forcesensor for robot,” in IEEE Sens. J. 18 (2018) 3632-3639.

[12] Minghua Luo, Xinhua Luo and Chunwei Pan, "A Stewart platformbased 3-axis force sensor for robot fingers," in 2011 Second International Conference on Mechanic Automation and Control Engineering, Hohhot, 2011, pp. 37-40.

[13] Y. Noh, J. Bimbo, S. Sareh, H. Wurdemann, J. Fras, D. S. Chathuranga, H. Liu, J. Housden, K. Althoefer and K. Rhode, "Multi-axis force/torque sensor based on simply-supported beam and optoelectronics", in Sensors 2016, vol. 16, no. 11, p.1936, 2016.

[14] A. Faragasso et al., "Novel uniaxial force sensor based on visual information for minimally invasive surgery," in 2014 IEEE International Conference on Robotics and Automation (ICRA), Hong Kong, 2014, pp. 1405-1410, doi: 10.1109/ICRA.2014.6907036.\#

[15] N. Bandari, J. Dargahi and M. Packirisamy, "Tactile Sensors for Minimally Invasive Surgery: A Review of the State-of-the-Art, Applications, and Perspectives," in IEEE Access, vol. 8, pp. 76827708, 2020, doi: 10.1109/ACCESS.2019.2962636.

[16] B. Ward-Cherrier, N. Pestell, L. Cramphorn, B. Winstone, M. E. Giannaccini, J. Rossiter, and N. F. Lepora, "The TacTip Family: Soft Optical Tactile Sensors with 3D-Printed Biomimetic Morphologies," Soft Robot., Jan. 2018.

[17] W. Yuan, S. Dong, and E. H. Adelson, "Gelsight: High-resolution robot tactile sensors for estimating geometry and force," in Sensors 17.12 (2017): 2762

[18] L.L. Nathans, F.L. Oswald and K. Nimon, "Interpreting multiple linear regression: A guidebook of variable importance", in Pract. Assess. Res. Eval. vol.17, pp. 1-19, 2012. 